\title{
ESTUDO DA RESPOSTA TERMICAMENTE ESTIMULADA DO COMPÓSITO LDPE/CB POR MEIO DA TÉCNICA DE CORRENTE DE DESPOLARIZAÇÃO TERMICAMENTE ESTIMULADA (TSDC)
}

\section{Edemir Luiz Kowalski*}

Departamento de Alta Tensão, Instituto de Tecnologia para o Desenvolvimento, Centro Politécnico, Universidade Federal do Paraná, CP 19067, 81531-990 Curitiba - PR, Brasil

Renê Robert

Departamento de Eletricidade, Universidade Federal do Paraná, Centro Politécnico, Universidade Federal do Paraná, CP 19067, 81531-990 Curitiba - PR, Brasil

Ademar Rúvolo Filho

Departamento de Química, Universidade Federal de São Carlos, CP 676, 13560-970 São Carlos -SP, Brasil

Recebido em 6/3/08, aceito em 17/12/08; publicado na web em 7/5/09

\begin{abstract}
STUDY OF RESPONSE THERMALLY STIMULATED OF LDPE/CB COMPOSITE BY OF THE TECHNIQUE OF THERMALLY STIMULATED DEPOLARIZATION CURRENT (TSDC). The thermally stimulated depolarization current (TSDC) in a range of temperature from 84 to $373 \mathrm{~K}$, has been applied to study the depolarization current of polyethylene and polyethylene composites in form of film and filled with commercial or oxidative surface treatment carbon black. The diagrams of TSDC obtained show that the composite in which the carbon black had received oxidative surface treatment reducing on an average depolarization current intensity in a magnitude order if compared to the composite with commercial carbon black. Therefore in the area between $\alpha$ and $\beta$ transitions the difference is accentuated by reaching a peak 55 times in a temperature of $240 \mathrm{~K}$. The difference in results is explained in terms of molecular interactions neighboring of carbon black particles.
\end{abstract}

Keywords: TSDC; LDPE; carbon black.

\section{INTRODUÇÃO}

Para a transmissão e distribuição de energia elétrica, são usados cabos que podem ser isolados ou protegidos, sendo que as camadas externa e semicondutora podem ser confeccionadas em material polimérico compósito. Normalmente se usa o polietileno de baixa densidade (LDPE) compósito com negro de fumo (NF). Estão sendo conduzidos estudos para identificar os mecanismos responsáveis pela condução elétrica nestes compósitos, ${ }^{1-8}$ porém, são raros os trabalhos em que se avalia o movimento molecular das cadeias poliméricas ativadas termicamente.

A distribuição e dispersão do NF na matriz polimérica são variáveis que influenciam diretamente as propriedades finais do compósito, sendo melhoradas com o tratamento superficial oxidativo do NF. Esta forma de tratamento melhora as propriedades de dispersão e distribuição do particulado na matriz polimérica. ${ }^{2,9-17}$ Neste trabalho são discutidos os mecanismos de condução elétrica e a mobilidade das cadeias poliméricas por meio da técnica de corrente de despolarização termicamente estimulada (TSDC) em amostras de LDPE com NF comercial e em LDPE com NF que sofreu tratamento superficial oxidativo.

\section{Revisão}

Quando o NF é adicionado ao polietileno (PE), o compósito resultante pode se tornar um semicondutor ou manter as propriedades de isolamento elétrico da matriz, dependendo somente da quantidade de NF adicionada. Se a adição for da ordem de $3 \mathrm{w} / \mathrm{w} \%$, a resistividade elétrica do polietileno não será alterada, porém, concentrações de NF superiores a este valor poderão aumentar a condutividade elétrica do compósito. ${ }^{1} \mathrm{~A}$ adição de concentrações até 3 w/w\% de NF dá ao compósito final uma proteção contra a degradação causada pela ação da radiação ultravioleta

*e-mail: edemir@lactec.org.br e a degradação térmica. ${ }^{1,-11}$ Estudos recentes têm enfatizado que o principal mecanismo de condução no compósito é o tunelamento de elétrons entre os agregados de NF, ou um efeito combinado de tunelamento entre agregados próximos com a percolação clássica. ${ }^{67}$ Percebe-se que todos os modelos de condução propostos explicam parcialmente os processos de condução em compósitos com NF, pois estes dependem fortemente de variáveis como distribuição, dispersão, estrutura do agregado, área superficial, tamanho e grau de cristalinidade da matriz polimérica. ${ }^{8}$ Alguns artigos têm enfatizado que o tempo de mistura afeta consideravelmente as propriedades condutivas do compósito. Esses estudos mostram que ao se aumentar o tempo de mistura ter-se-á um particulado mais homogeneamente distribuído na matriz, causando uma redução na condutividade volumétrica do compósito final. ${ }^{2,18}$

A condutividade elétrica é um processo termicamente ativado, e segue a lei de Arrhenius:

$$
\sigma=\sigma_{0} \exp \left(-\frac{\Delta E}{k T}\right)
$$

onde $\Delta E$ representa a energia de ativação para o processo de condução, $k$ é a constante de Boltzmann e $\sigma_{0}$ é um fator pré-exponencial. ${ }^{3,12} \mathrm{~A}$ condutividade elétrica depende fortemente das interfaces amorfo-cristalina e amorfo-agregado. ${ }^{3}$ As interfaces representam barreiras de potencial que deverão ser transpostas pelos portadores de carga elétrica e a presença dos agregados altera a distribuição e acumulação de carga elétrica alterando, assim, a condutividade elétrica final do compósito.

A interação entre os agregados do NF com a matriz polimérica limita-se fundamentalmente a interações superficiais, podendo ser de origem física ou química, assunto este que gera muita controvérsia, de forma tal que estes fenômenos ainda não são bem compreendidos. Quanto maior a área superficial do NF, maior será o número de interações entre a matriz e o particulado. Assim, as partes da cadeia que interagem com o NF possuem movimento restrito. Esta restrição de 


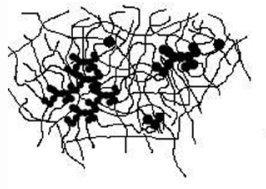

a

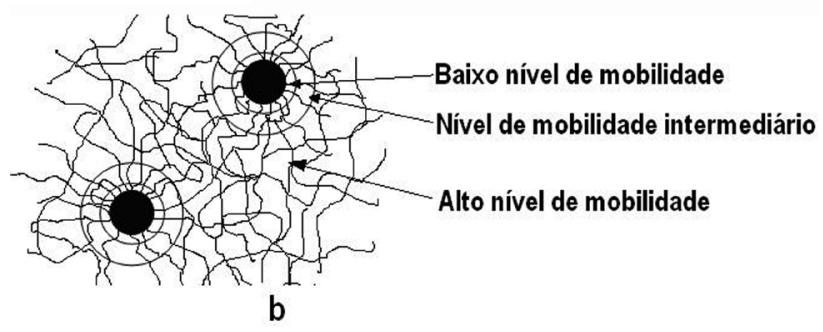

Figura 1. Em (a) um modelo para as interações moleculares com as partículas aglomerados e agregados de NF. Em (b) os possíveis níveis de interação entre a cadeia principal e o NF, onde se apresentam três possíveis níveis de mobilidade

movimento está ligada a fatores tais como área superficial específica, estrutura e superfície do NF e tipo de matriz. Com o tratamento térmico, ter-se-á um crescimento de cristalitos na superfície pela incorporação dos aglomerados do NF que estavam na fase amorfa. ${ }^{1}$ Devido às interações interfaciais entre as cadeias poliméricas e NF, tem-se diferentes níveis de mobilidade molecular próximos à superfície do agregado de NF, onde a região sobre a superfície tem um nível de mobilidade reduzido, a região seguinte um nível de mobilidade intermediário e as regiões afastadas do agregado uma mobilidade maior, como é mostrado na Figura $1 .{ }^{19}$ Sweitzer e colaboradores sugerem que as interações entre matriz e NF tratado superficialmente são consideravelmente maiores que nos casos onde o NF é comum. ${ }^{20}$

\section{Corrente de despolarização termicamente estimulada (TSDC)}

A aplicação da técnica do TSDC em materiais dielétricos pode fornecer informações sobre perdas dielétricas, constante dielétrica e mecanismos de relaxação dielétrica e mecânica e condução em faixas de freqüências não atingidas por outras técnicas de análise de dielétricos. Em função da sensibilidade desta técnica, movimentos moleculares não observados por outras técnicas passam a ser perceptíveis..$^{21-28}$

$\mathrm{O}$ experimento de corrente termicamente estimulada baseia-se na colocação do material a ser estudado entre duas placas condutoras paralelas (eletrodos), criando assim um capacitor plano de placas paralelas. No experimento, aplica-se um campo elétrico $\vec{E}$, contínuo com a amostra a uma temperatura de polarização $\mathrm{T}_{\mathrm{p}}$, durante um tempo de polarização $\mathrm{t}_{\mathrm{p}}$. A seguir, a amostra sob a ação do campo elétrico é resfriada até a temperatura final $\mathrm{T}_{0}$, isotermicamente é colocada em curto-circuito, para que sofra uma despolarização inicial onde são eliminadas as correntes elétricas transientes até que seja atingida uma corrente elétrica da ordem de $10^{-14} \mathrm{~A}$, quando passa a ser aquecida a uma taxa constante $\mathrm{h}$, até uma temperatura final $\mathrm{T}_{\mathrm{f}}$. $\mathrm{O}$ aquecimento faz com que surja uma corrente elétrica $\mathrm{i}(\mathrm{T})$ em função da temperatura medida no circuito externo. Essa corrente elétrica registrada como uma função da temperatura resulta no chamado termograma de TSDC. ${ }^{29}$ Por meio do termograma, obtêm-se informações tais como energia de ativação e tempo de relaxação dos mecanismos termicamente ativados, bem como número de dipolos que foram polarizados durante o processo.

\section{PARTE EXPERIMENTAL}

\section{Materiais usados}

Dois tipos de filmes comerciais de LDPE com espessura de 160 $\mu \mathrm{m}$ foram fornecidos pela Union Carbide do Brasil, sendo o primeiro puro e o segundo um material compósito com um índice de NF em torno de $3 \mathrm{w} / \mathrm{w} \%$. Também foi usado o LDPE MN 379 Poliolefinas em forma de pó para preparo de um compósito onde o NF N 220 sofreu tratamento oxidativo superficial prévio. O grau de cristalinidade obtido, usando-se difratometria de Raios-X, foi de $31 \%$ para o composto comercial e $32 \%$ para o LDPE puro. Com a técnica de DSC, as temperaturas de fusão e transição vítrea foram, respectivamente, de 383 e 196 K para duas amostras.

\section{Tratamento do negro de fumo}

O negro de fumo da Union Carbide N 220 foi misturado com ácido nítrico sob refluxo a uma temperatura de $373 \mathrm{~K}$ em uma mistura de $\mathrm{HNO}_{3} / \mathrm{NF}$ de $7: 1 / \mathrm{m}: \mathrm{m}$, seguido de lavagem com água destilada até obter-se $\mathrm{pH}$ neutro e, posteriormente, secado a $323 \mathrm{~K}$ por 4 dias.

\section{Compósito LDPE/NF}

O compósito LDPE MN 379 com 3 w/w\% de NF tratado superficialmente foi preparado por meio de um misturador Daris, que é constituído por uma câmara cilíndrica contendo uma haste horizontal onde estão fixadas 6 pás. A fusão do polímero ocorre por dissipação de energia viscosa. $\mathrm{O}$ ponto final de mistura adotado foi de 1 min após a corrente passar pelo Wet Point. Após a mistura, foram confeccionados por termoprenssagem filmes do compósito com espessura média de160 $\mu \mathrm{m}$. Usando as mesmas técnicas descritas no item anterior, o grau de cristalinidade obtido para este compósito foi de $36 \%$ e a temperatura de transição vítrea e fusão, respectivamente, de 195 e 383 K.

As amostras utilizadas nos experimentos de TSC tiveram depositados eletrodos de alumínio sobre as duas superfícies, de espessura aproximada de $70 \mu \mathrm{m}$ e diâmetro de $40 \mathrm{~mm}$, através do processo de evaporação do alumínio em alto vácuo, utilizando-se a Metalizadora da Edwards ${ }^{\circledR}$.

As amostras passaram pelo processo de pré-condicionamento, antes de serem realizados os experimentos de despolarização termicamente estimulada. O pré-condicionamento consiste em aplicar-se um campo elétrico de mesma intensidade ao usado no experimento de TSDC durante $8 \mathrm{~h}$ e, em seguida, realiza-se a despolarização da amostra promovendo-se o curto circuito entre os eletrodos.

O equipamento de TSDC utilizado é uma câmara de criogenia que trabalha entre as temperaturas de 75 e $473 \mathrm{~K}$ e opera com vácuo da ordem de $10^{-7}$ torr. Para a leitura da corrente elétrica é usado um eletrômetro Keithley modelo 617. O controle de temperatura é feito por meio de um controlador de temperatura Gefran modelo 330 e os dados são adquiridos através de computador.

A Figura 2 mostra o termograma comparativo entre o compósito comercial e o compósito onde o NF sofreu tratamento superficial oxidativo. Percebe-se que entre 84 e $170 \mathrm{~K}$ os compósitos apresentam um comportamento semelhante.

Segundo Iedae colaboradores, para temperaturas abaixo e próximas a $103 \mathrm{~K}$, prevalecem os saltos de portadores de carga em função de interações coulombicas. ${ }^{30}$ Próximo a $200 \mathrm{~K}$, percebe-se considerável aumento da densidade de corrente de despolarização, principalmente no compósito comercial. Nesta temperatura tem-se uma transição vítrea do material: ocorre um aumento do volume livre da matriz do compósito, onde curtos movimentos segmentais da cadeia principal em regiões de interface associadas a cristalitos, rotação envolvendo de 1 a 7 ligações (virabrequim), movimentos micro-brownianos das 


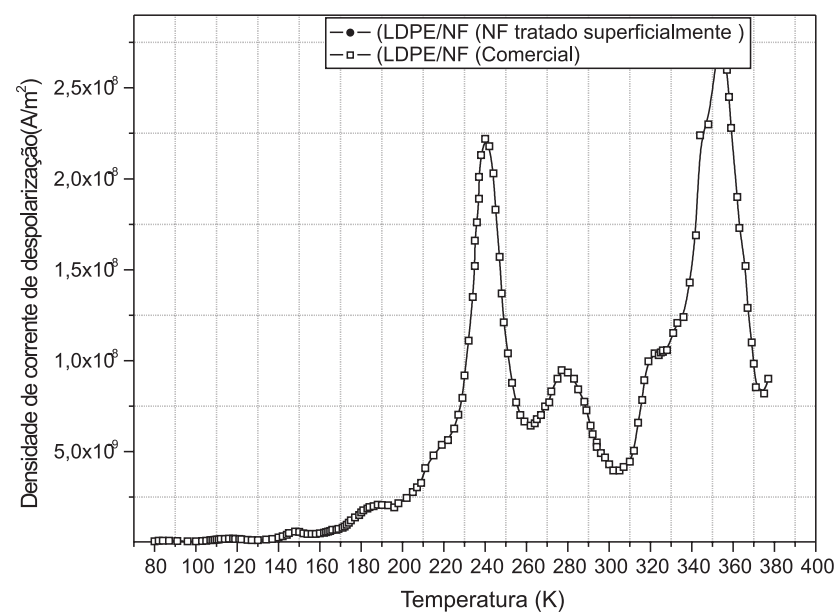

Figura 2. Termogramas comparativos para os compósitos com NF, onde $t_{p}$ $=1 \mathrm{~h}, \mathrm{E}=11,6 \mathrm{MV} / \mathrm{m}$ e $T_{p}=323 \mathrm{~K}$

fases amorfas, movimento de portadores de cargas elétricas presos a armadilhas, em bandas de valência ou condução, são os possíveis mecanismos responsáveis pela resposta do TSDC. . $^{21,31,32}$

Entre 196 e aproximadamente $263 \mathrm{~K}$, observa-se que as diferenças entre as correntes de despolarização chegam em média a 10 vezes, como pode ser visto nas Figuras 2 e 3, atingindo um máximo em aproximadamente $248 \mathrm{~K}$. Nessa região tem-se a manifestação dos mecanismos ligados à transição $\beta$, atribuídos ao movimento da cadeia principal, pontos de ramificação, grupos laterais, bem como portadores de cargas presos em armadilhas., ${ }^{2,18,21}$

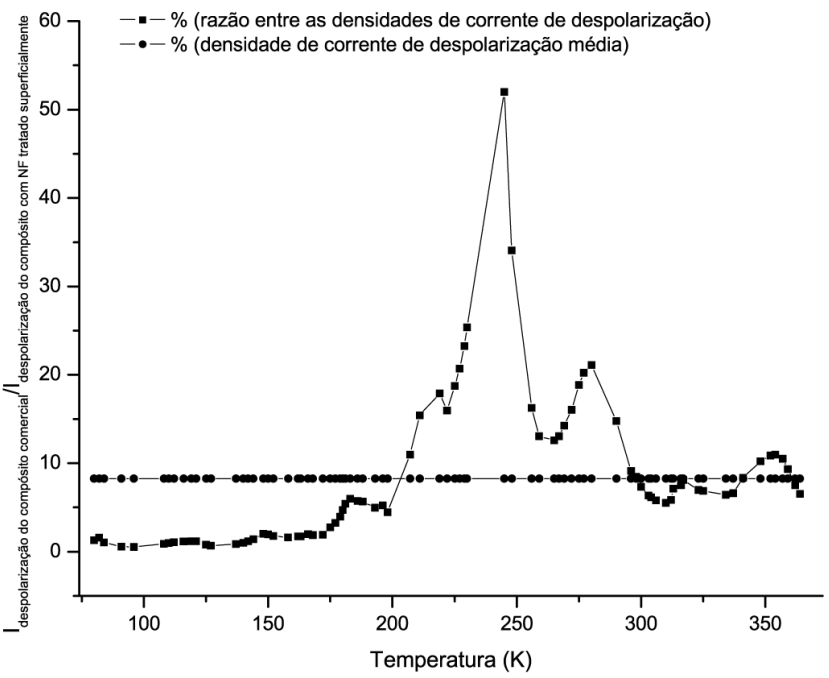

Figura 3. Razão entre as correntes de despolarização do compósito comercial e do compósito que sofreu tratamento superficial oxidativo

Acima de $320 \mathrm{~K}$ surgem as respostas ligadas às transições $\alpha \mathrm{e}$ $\alpha$, que se referem aos movimentos das regiões amorfas da matriz e movimentos nos contornos de grãos, como também ao surgimento das respostas ligadas ao movimento de cargas elétricas espaciais, possivelmente injetadas pelos eletrodos durante o processo de polarização das amostras, além de relaxações dipolares. ${ }^{32,35-38}$

Como se pode perceber, a resposta à despolarização termicamente estimulada do compósito comercial é em média uma ordem de grandeza maior que a do compósito que sofreu tratamento superficial oxidativo. Com exceção da região abaixo de $196 \mathrm{~K}$, todas as demais regiões apresentaram uma densidade de corrente de despolarização bastante diferenciada. As diferenças conformacionais entre as amos- tras limitam-se à forma de mistura do particulado à matriz, bem como ao tratamento superficial oxidativo. Com relação ao tempo de mistura, obtém-se um compósito com melhor distribuição e dispersão dos agregados de NF na matriz, ${ }^{2,18,21}$ e a melhor distribuição e dispersão, como pode ser visto na Figura 4, e o tratamento superficial oxidativo também melhoram a qualidade da interface entre os agregados e a matriz, facilitando a sua dispersão e distribuição.

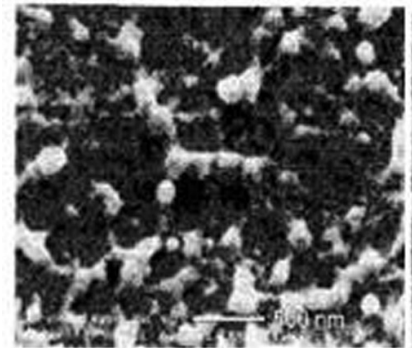

a

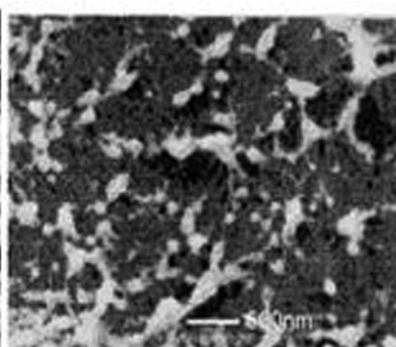

b
Figura 4. Microscopia eletrônica de varredura, onde as partes brancas são os agregados de NF e as partes escuras a matriz polimérica; em (a), o compósito comercial onde o NF tem tamanho médio de $210 \mathrm{~nm}$; em (b), o compósito onde o NF sofreu tratamento superficial oxidativo com tamanho médio de $100 \mathrm{~nm}$

Os mecanismos que respondem ao TSDC para amostras de LDPE são bastante discutidos, porém o mecanismo que responde em $240 \mathrm{~K}$ no compósito comercial chama bastante a atenção. No termograma feito sobre as matrizes, como pode ser visto na Figura 5, esta resposta não aparece e no compósito que sofreu o tratamento superficial oxidativo é pouco pronunciada. Estes resultados permitem supor que a resposta está associada à presença do NF. A razão entre as correntes de despolarização do compósito comercial e o compósito com o NF que sofreu tratamento superficial oxidativo chega à ordem de 10 vezes em toda a faixa de temperatura e, no pico em $240 \mathrm{~K}$, a 55 vezes como pode ser observado na Figura 3. Estes resultados permitem assumir a hipótese de que a diferença pode estar associada a: melhor distribuição e dispersão do NF em função da forma de mistura e, ao tratamento superficial oxidativo. Com relação à melhor distribuição e dispersão do NF na matriz, como pode ser observado na Figura 4, percebe-se uma redução no tamanho médio das partículas, sugerindo um aumento na área superficial das mesmas, na molhabilidade e no contato com segmentos das cadeias da fase amorfa da matriz de LDPE.

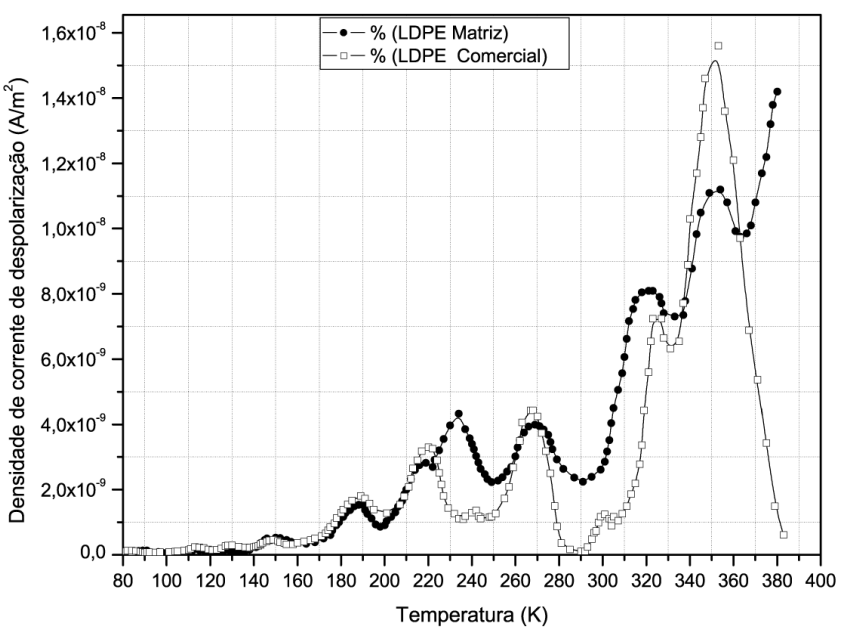

Figura 5. Termogramas comparativos para as matrizes de LDPE, onde tp $=$ $1 \mathrm{~h}, \mathrm{E}=11,6 \mathrm{MV} / \mathrm{m}$ e $T p=323 \mathrm{~K}$ 
O tratamento superficial oxidativo aumenta o número de grupos funcionais contendo oxigênio, modificando a natureza neutra ou básica da superfície para ácida, além de eliminar a presença de poros nos agregados de NF. Na temperatura de $240 \mathrm{~K}$, as respostas do TSDC são associadas à relaxação da fase amorfa do LDPE e devidas aos movimentos segmentais localizados, e sua intensidade pode estar associada ao grau de cristalinidade do polímero, onde polímeros mais cristalinos apresentam respostas menos intensas. ${ }^{39}$ Como discutido na introdução, para NF com grande área superficial o número de interações entre a matriz e a fase amorfa do polímero aumenta muito, principalmente quando o NF sofre tratamento superficial oxidativo. ${ }^{39}$ Nestes casos tem-se diferentes regiões de mobilidade dos segmentos das cadeias poliméricas em torno dos agregados de partículas de NF, como mostra a Figura 1. Portanto, é razoável assumir que no compósito com NF comercial as interações provavelmente são menos intensas que aquelas do compósito com NF que sofreu tratamento superficial, sendo que para a mesma energia térmica fornecida pelo TSDC, obtém-se um grau de despolarização menos intenso, devido aos movimentos segmentais reduzidos para a amostra onde o compósito sofreu tratamento superficial oxidativo. Com relação ao grau de cristalinidade das amostras, estes não apresentam diferença substancial para interferir fortemente nas respostas.

Em termos de respostas elétricas, mecanismos como polarização interfacial, condução através de armadilhas e defeitos estruturais, processos de tunelamento inter-agregado podem se tornar pronunciados. $\mathrm{O}$ tratamento superficial oxidativo melhora a qualidade da interface matriz agregado, reduzindo as imperfeições e o número de armadilhas e efeitos de polarização interfacial. Assim, ocorre uma redução no número de portadores de carga capturados pelas armadilhas injetados pelos eletrodos durante o processo de polarização da amostra. Como há uma melhor dispersão e distribuição dos agregados pela matriz, possíveis processos de condução por tunelamento inter-agregados tornam-se menos intensos. ${ }^{3,5-7}$

\section{CONCLUSÃO}

Os resultados obtidos mostram que o tratamento superficial oxidativo e $\mathrm{a}$ forma de mistura do NF à matriz influenciam fortemente sobre os mecanismos que respondem ao serem termicamente ativados no TSDC. $\mathrm{O}$ tratamento superficial oxidativo melhora a qualidade da interface matriz-agregado, reduzindo os movimentos de escala molecular nas diferentes regiões de mobilidade dos segmentos das cadeias poliméricas em torno dos agregados de partículas de NF. À temperatura de $240 \mathrm{~K}$ é observada uma resposta no TSDC, que é atribuída aos movimentos moleculares da cadeia da matriz polimérica em torno do agregado de NF. A mobilidade dos portadores de carga elétrica é reduzida, bem como os efeitos de polarização interfacial, em função de uma melhora na distribuição e dispersão dos agregados pela matriz amorfa.

\section{MATERIAL SUPLEMENTAR}

O material suplementar, disponível em http://quimicanova.sbq.org. br, na forma de arquivo PDF, com acesso livre, contém os termogramas de três amostras diferentes do compósito comercial e do compósito onde o NF sofreu tratamento superficial oxidativo, além dos termogramas obtidos para as matrizes poliméricas de PE comercial e a matriz do compósito que recebeu o NF tratado superficialmente respectivamente. Estes termogramas mostram a repetibilidade dos resultados experimentais.

\section{REFERÊNCIAS}

1. Ramos, R. J.; IEEE Transitions on Dielectrics and Electrical Insulation 2000, 7, 855 .

2. Dilhan, M. K.; Birinci, E.; Yazici, R.; Karuv, B.; Walsh, S.; Polym. Eng.
Sci. 2002, 42, 1609.

3. Brosseau, C.; Boulic, F.; Berounal, A.; IEEE Conference Record of the IEEE International Symposium on Electrical Insulation, Montreal, Canada, 1996.

4. http://acs.omnibooksonline.com/data/papers/1995_732.pdf, acessada em Setembro 2007.

5. Xi, B.; Chen, G.; IEEE Proceedings of $6^{\text {th }}$ International Conference on Properties and Applications of Dielectric Materials, Xi'an Jiaotong University Xi'an China, 2000.

6. Xi, B.; Chen, G.;. IEEE Proceedings of $7^{\text {th }} I C S D$, Eidhoven, Netherlands, 2001.

7. Peña, J. M.; Allen, N. S.; Edge, M.; Liauw, C. M.; Valange, B.; Polym. Degrad. Stab. 2001, 72, 259.

8. Blow, C. M.; Polymer 1973, 14, 309

9. Phillips, P. L.; IEEE Transitions on Dielectrics and Electrical Insulation 1978, 13, 69 .

10. Mayoux, C.; IEEE Transitions on Dielectrics and Electrical Insulation 1997, 4, 665 .

11. Barlow, A.; IEEE Electrical Insulation Magazine 1991, 7, 9.

12. Hugues, S. O.; IEEE Electrical Insulation Magazine 1976, 11, 1.

13. Margolis, J. M.; Conductive Polymers and Plastics, Chapman \& Hall: New York, 1989.

14. Delmonte, J .; Metal and Polymer Composites, Van Nostrand: New York, 1990.

15. Bigg, D. M.; Polym. Compos. 1986, 7, 125.

16. Bigg, D. M.; Bhattacharya, S.; Metal Filled Polymers, Marcel Dekker: New York, 1986.

17. Yu, G. ; Zhang, Q.; Zeng, H.; J. Appl. Polym. Sci. 1998, 70, 559.

18. Cheah, K.; Forsyth, M.; Simon, G.; Synth. Met. 1999, 102, 1232.

19. Kaufman, S.; Slichter, W. P.; Davis, D. D.; J. Appl. Polym. Sci. 1971, 9, 829.

20. Sweitzer ,C. W.; Rubber Age. 1952, 72, 55.

21. Das-Gupta, D. K.; J. Electrostatics 2001, 51, 159.

22. Gross, B.; Endevouar. 1971, 30, 115.

23. Goitiandia, L.; Alegria, A.; J. Non-Cryst. Solid. 2001, 287, 237.

24. Nogales, A.; Sauer, B. B.; J. Polym. Sci., Part B: Polym. Phys. 1998, 36 , 913.

25. Galop, M.; J. Therm. Anal. Calorim. 1997, 49, 107.

26. Mano, J. F.; Mendez, S. L.; J. Appl. Phys. 2001, 89, 1844.

27. Brostow, W.; D’Souza, A.; Annual Technical Conference of the Society of Plastics Engineers, Toronto, Canada, 1997.

28. Neagu, E. R.; Marat-Mendes, J. N.; Neagu, M. R.; Das-Gupta, D. K.; J. Appl. Phys. 1999, 85, 2330.

29. Sessler, G. M.; Breazeale, M. A.; Electrets, $2^{\text {nd }}$ ed., Springer-Verlag: Berlin, 1987.

30. Ieda, E.; Cai, G.; Suzuoki, Y.; Mizutani, T.; Memoires of the Faculty of Engieneering, Nagoya University 1986, 38, 137.

31. Mizutani, T.; Susuoki, Y.; Ieda, M.; J. Appl. Phys. 1977, 48, 2408.

32. Laredo, E.; Suarez, N.; Bello, A.; Márquez, L.; J. Polym. Sci., Part B: Polym. Phys. 1996, 34, 641.

33. Das- Gupta, D. K.; Duffy. J. S.; J. Electrostatic. 1983, 14, 99.

34. MacCrum, N. G.; Read, B. E.; Willians, G.; Anelastic and Dielectric Effects in Polymeric Solid, John Wiley \& Sons Ltd: New York, 1967.

35. Stachurski, Z.; Ward, H.; J. Polym. Sci., Part B: Polym. Phys. 1968, 6 , 1817.

36. Tanaka, A.; Chang, E. P.; Delf, B.; Kimura, I.; Stein, R. S.; J. Polym. Sci., Part B: Polym. Phys. 1973, 11, 1891

37. Mizutani, T.; Tsukahara, T.; Ieda, M.; Jpn. J. Appl. Phys., Part 2 1980, 19,2095

38. Sawa, G.; Kawade, M.; Ieda, M.; Jpn. J. Appl. Phys., Part 2 1974, 13, 1574.

39. Kalika, D. S.; Handbook of Low and High Dielectric Constant Materials and Their Applications, Academic Press: New York, 1999, cap. 7. 


\section{ESTUDO DA RESPOSTA TERMICAMENTE ESTIMULADA DO COMPÓSITO LDPE/CB POR MEIO DA TÉCNICA DE CORRENTE DE DESPOLARIZAÇÃO TERMICAMENTE ESTIMULADA (TSDC)}

\section{Edemir Luiz Kowalski*}

Departamento de Alta Tensão, Instituto de Tecnologia para o Desenvolvimento, Centro Politécnico, Universidade Federal do Paraná, CP 19067, 81531-990 Curitiba - PR, Brasil

Renê Robert

Departamento de Eletricidade, Universidade Federal do Paraná, Centro Politécnico, Universidade Federal do Paraná, CP 19067, 81531-990 Curitiba - PR, Brasil

Ademar Rúvolo Filho

Departamento de Química, Universidade Federal de São Carlos, CP 676, 13560-970 São Carlos -SP, Brasil

As Figuras 1S e 2S mostram três termogramas obtidos com três amostras diferentes do compósito comercial e do compósito onde o NF sofreu tratamento superficial oxidativo, respectivamente. As Figuras 3S e 4S mostram os termogramas obtidos para as matrizes poliméricas de PE comercial e a matriz do compósito que recebeu o NF tratado superficialmente, respectivamente. Os termogramas mostram a repetibilidade dos resultados experimentais.

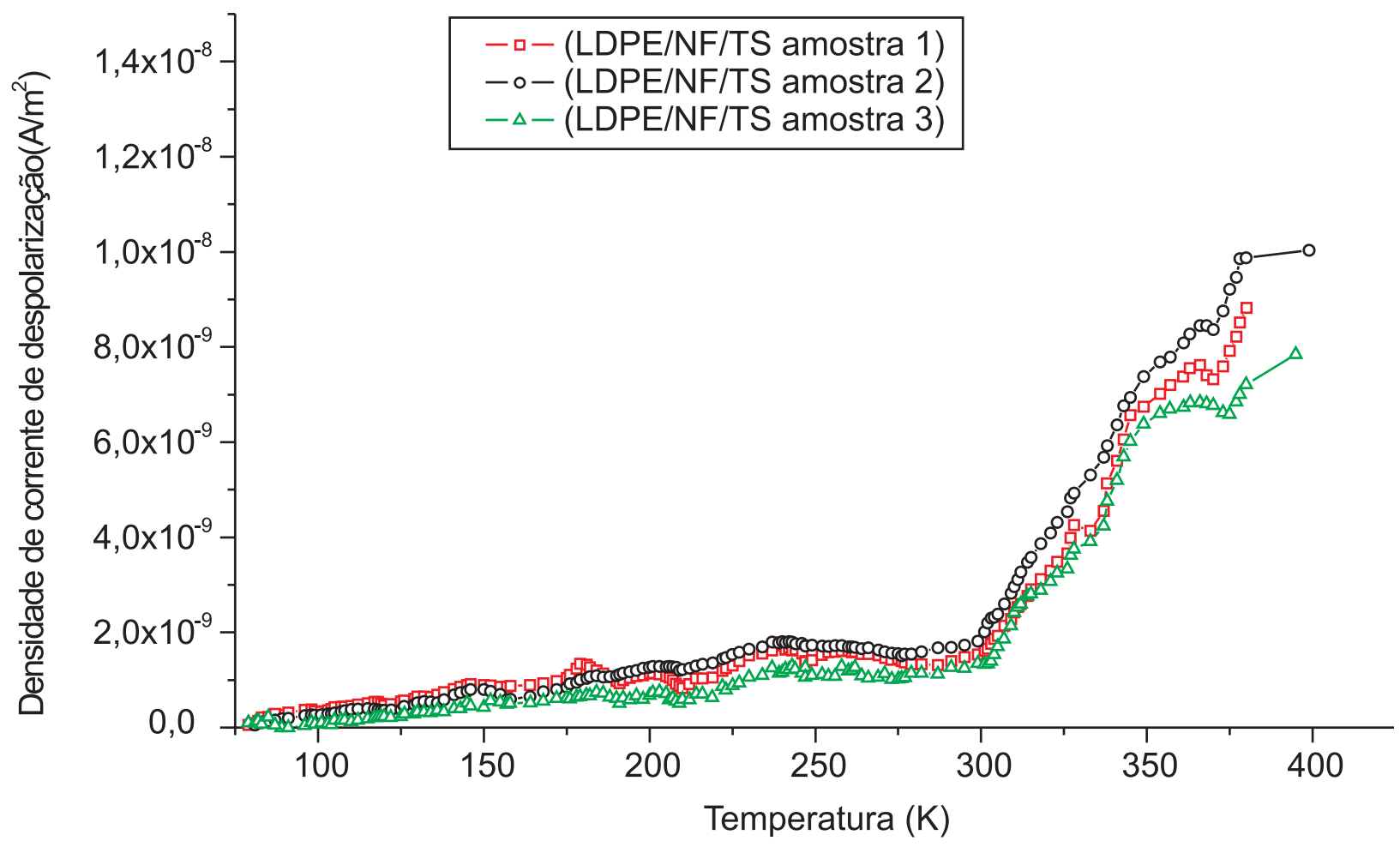

Figura 1S.Termogramas obtidos para o compósito comercial 


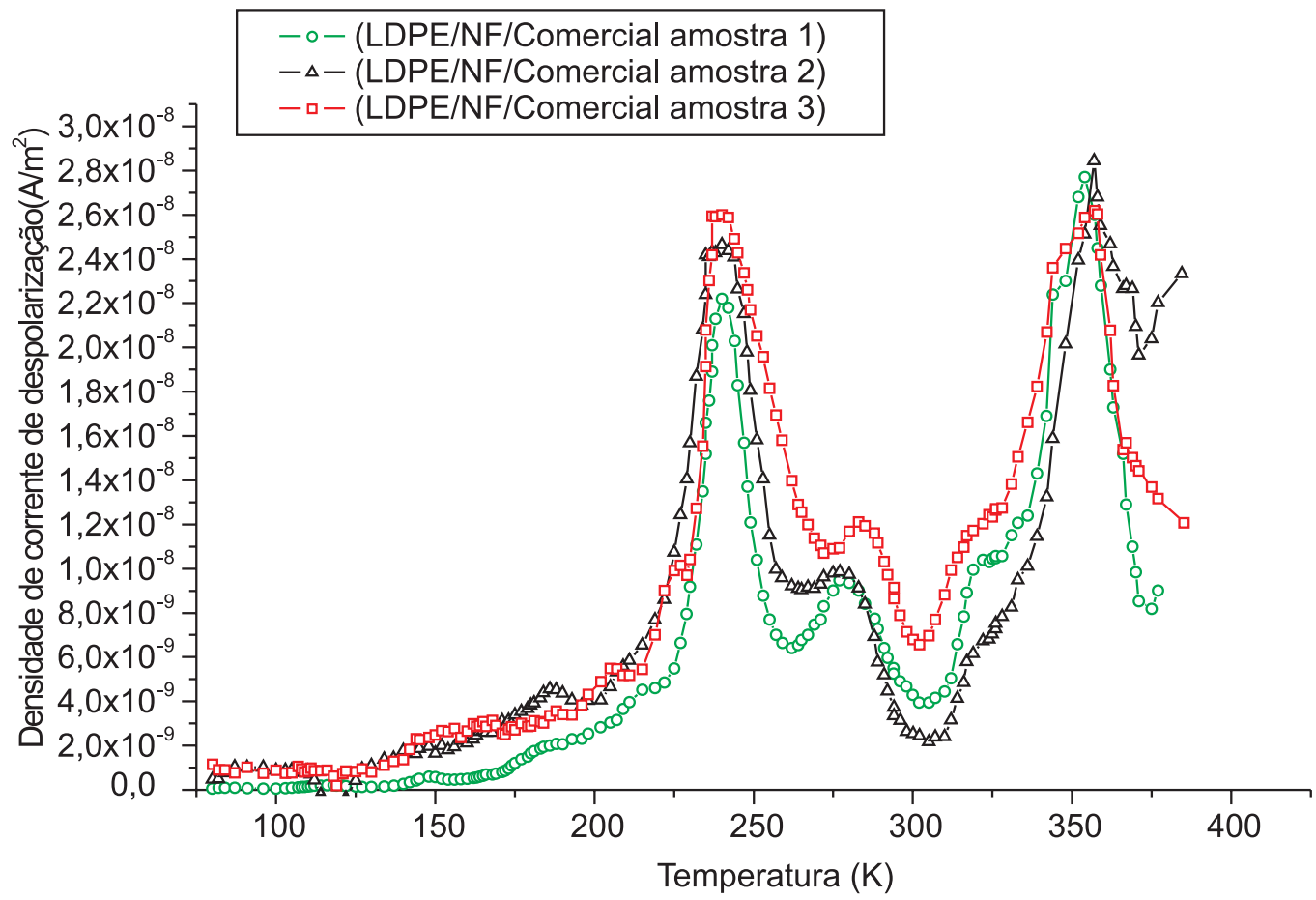

Figura 2S.Termogramas obtidos para os compósitos onde o NF sofreu tratamento superficial oxidativo

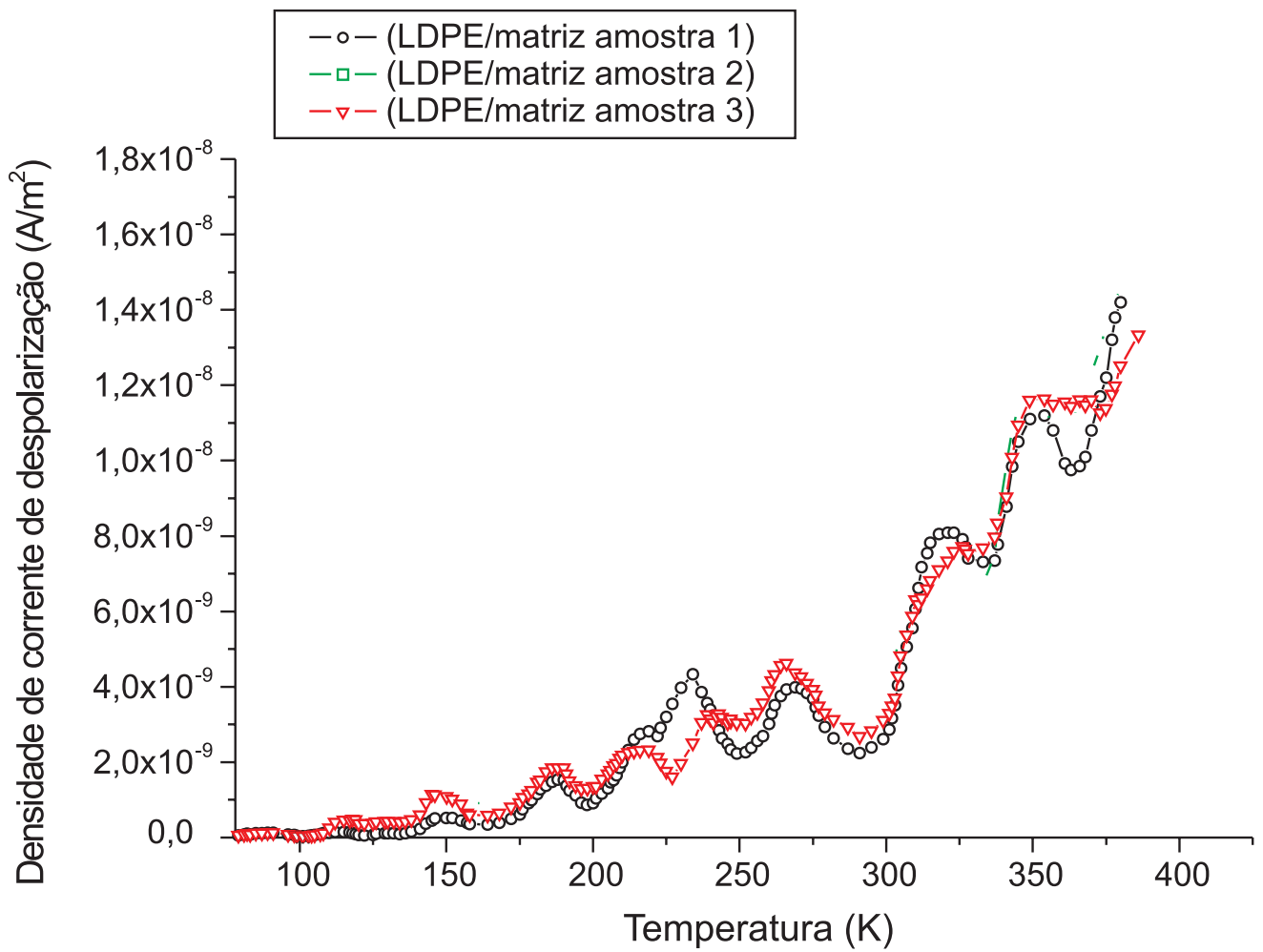

Figura 3S. Termogramas obtidos para três matrizes de PE comercial 


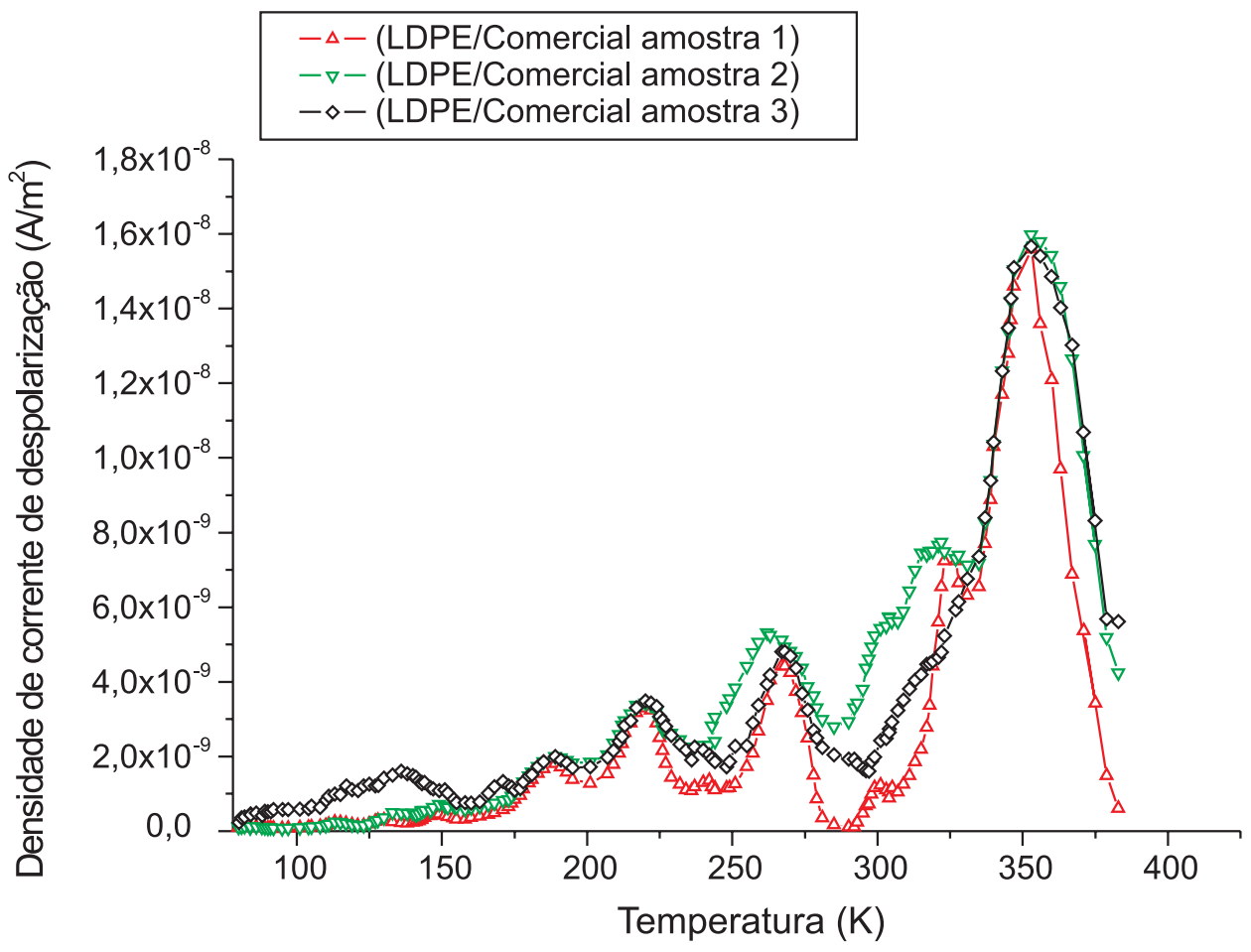

Figura 4S. Termogramas obtidos para três matrizes usadas no compósito onde o NF sofreu tratamento 